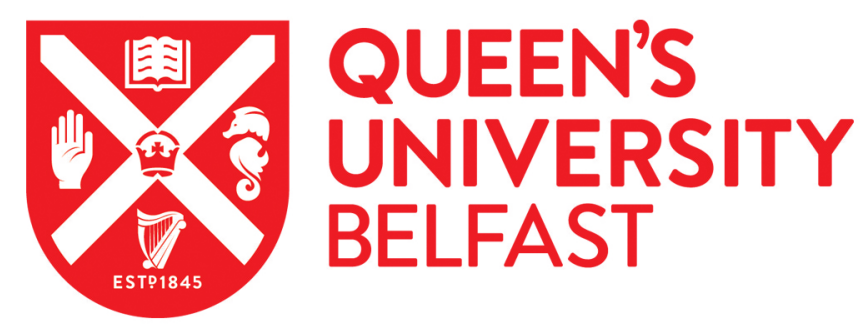

\title{
Relativistic R-matrix calculations for the electron-impact excitation of neutral molybdenum
}

\author{
Smyth, R., Johnson, C., Ennis, D., Loch, S., Ramsbottom, C., \& Ballance, C. (2017). Relativistic R-matrix \\ calculations for the electron-impact excitation of neutral molybdenum. Physical Review A (Atomic, Molecular, \\ and Optical Physics), 96(4), [042713]. https://doi.org/10.1103/PhysRevA.96.042713
}

Published in:

Physical Review A (Atomic, Molecular, and Optical Physics)

Document Version:

Publisher's PDF, also known as Version of record

Queen's University Belfast - Research Portal:

Link to publication record in Queen's University Belfast Research Portal

\section{Publisher rights}

Copyright 2017 American Physical Society. This work is made available online in accordance with the publisher's policies. Please refer to any applicable terms of use of the publisher. .

\section{General rights}

Copyright for the publications made accessible via the Queen's University Belfast Research Portal is retained by the author(s) and / or other copyright owners and it is a condition of accessing these publications that users recognise and abide by the legal requirements associated with these rights.

Take down policy

The Research Portal is Queen's institutional repository that provides access to Queen's research output. Every effort has been made to ensure that content in the Research Portal does not infringe any person's rights, or applicable UK laws. If you discover content in the Research Portal that you believe breaches copyright or violates any law, please contact openaccess@qub.ac.uk. 


\title{
Relativistic $\boldsymbol{R}$-matrix calculations for the electron-impact excitation of neutral molybdenum
}

\author{
R. T. Smyth, ${ }^{1, *}$ C. A. Johnson, ${ }^{2}$ D. A. Ennis, ${ }^{2}$ S. D. Loch, ${ }^{2}$ C. A. Ramsbottom, ${ }^{1}$ and C. P. Ballance ${ }^{1}$ \\ ${ }^{1}$ School of Mathematics and Physics, Queen's University Belfast, Belfast BT7 1NN, Northern Ireland, United Kingdom \\ ${ }^{2}$ Department of Physics, Auburn University, Auburn, Alabama 36849, USA \\ (Received 30 July 2017; published 18 October 2017)
}

\begin{abstract}
A recent PISCES-B Mod experiment [Nishijima et al., J. Phys. B 43, 225701 (2010)] has revealed up to a factor of 5 discrepancy between measurement and the two existing theoretical models [Badnell et al., J. Phys. B 29, 3683 (1996); Bartschat et al., J. Phys. B 35, 2899 (2002)], providing important diagnostics for Mo I. In the following paper we address this issue by employing a relativistic atomic structure and $R$-matrix scattering calculations to improve upon the available models for future applications and benchmark results against a recent Compact Toroidal Hybrid experiment [Hartwell et al., Fusion Sci. Technol. 72, 76 (2017)]. We determine the atomic structure of Mo I using GRASP ${ }^{0}$, which implements the multiconfigurational Dirac-Fock method. Fine structure energies and radiative transition rates are presented and compared to existing experimental and theoretical values. The electron-impact excitation of Mo I is investigated using the relativistic $R$-matrix method and the parallel versions of the Dirac atomic $R$-matrix codes. Electron-impact excitation cross sections are presented and compared to the few available theoretical cross sections. Throughout, our emphasis is on improving the results for the $z^{5} P_{1,2,3}^{o} \rightarrow$ $a^{5} S_{2}, z^{7} P_{2,3,4}^{o} \rightarrow a^{7} S_{3}$ and $y^{7} P_{2,3,4}^{o} \rightarrow a^{7} S_{3}$ electric dipole transitions of particular relevance for diagnostic work.

DOI: 10.1103/PhysRevA.96.042713
\end{abstract}

\section{INTRODUCTION}

Atomic physics relies heavily upon the interplay between the interpretation of experimental measurement and the theoretical calculation that underpins it. Experiment may reveal deficiencies or inconsistencies between previous theoretical models, as is the case for neutral molybdenum, for which the following important diagnostic lines,

(i) $z^{5} P_{1,2,3}^{o} \rightarrow a^{5} S_{2}(557.0 \mathrm{~nm}, 553.3 \mathrm{~nm}, 550.6 \mathrm{~nm})$,

(ii) $z^{7} P_{2,3,4}^{o} \rightarrow a^{7} S_{3}(390.3 \mathrm{~nm}, 386.4 \mathrm{~nm}, 379.8 \mathrm{~nm})$,

(iii) $y^{7} P_{2,3,4}^{o} \rightarrow a^{7} S_{3}(319.4 \mathrm{~nm}, 317.0 \mathrm{~nm}, 313.3 \mathrm{~nm})$,

exhibit a factor of 2-5 difference with theoretical prediction. The main purpose of the following paper is to resolve this discrepancy, identify other diagnostic lines that have a strong temperature and density dependence, and validate our calculations against a recent Compact Toroidal Hybrid (CTH) experiment [1] using extensive collisional-radiative modeling.

Small-scale calculations for neutral molybdenum in $L S$ coupling have been carried out [2,3] using nonrelativistic $R$-matrix theory. However, these models are incomplete, including only 6 and 67 terms, respectively, in the scattering calculations, directly leading to inaccuracies in any work related to modeling using Mo I. It is evident that further work must be carried out using large-scale scattering calculations and a much more comprehensive atomic structure than what currently exists to improve upon existing models, rectifying the differences that currently exist between theory and experiment for applications to future problems. The results discussed throughout have wider implications in areas such as plasma physics, where neutral molybdenum is extensively used as a plasma facing component (PFC) in tokamaks.

Experimental investigations using Mo I PFCs have been carried out at Alcator C-Mod [4-6]; PISCES-B Mod [7,8]; and the DIII-D tokamak [9]. Furthermore, NSTX-U has plans to install a limited set of molybdenum tiles, with the potential

\footnotetext{
*rsmyth41@qub.ac.uk
}

to upgrade to a fully high- $Z$ wall [10]. However, a major disadvantage of using high- $Z$ atoms such as molybdenum $(Z=42)$ lies in the fact that they have the potential to greatly hinder the performance of the tokamak, arising due to an influx of impurities from the PFCs into the fusion plasma core. Such an occurrence is highly undesirable, since only low concentrations of Mo I can be tolerated within the plasma ( $\sim 0.1 \%$ compared to a concentration of $\sim 10 \%$ of carbon [11]) before thermonuclear fusion is quenched. Thus, this influx of impurities must be accurately characterized, requiring electron-impact excitation, electron-impact ionization, and radiative transition data [12], the precision of which is not offered by existing models.

This paper critically assesses the current models for Mo I and presents updated electron-impact excitation and atomic structure data using a fully relativistic approach culminating in the largest $R$-matrix scattering calculation for neutral molybdenum to date. In the next section we present the atomic structure calculations using the multiconfigurational Dirac-Fock program GRASP ${ }^{0}[13,14]$. Fine structure energies and radiative transitions rates are presented and the results are compared to experimental values where available. In Sec. III we present the details and results of the electron-impact excitation calculations using the most recent versions of the Dirac atomic $R$-matrix codes (DARC) [15]. Excitation cross sections for important transitions are presented and compared to the few available theoretical cross sections. In Sec. IV we briefly discuss the theory and results of our population modeling calculations and benchmark our results against new data obtained from a recent CTH experiment. Finally, in Sec. V, we present our conclusions and discuss future calculations involving Mo I.

\section{ATOMIC STRUCTURE}

First, we must note the difficulty of modeling such a complex atom due to the presence of half-open $4 d$ subshells in its ground state (ground-state configuration of $4 d^{5} 5 s$ ) and 


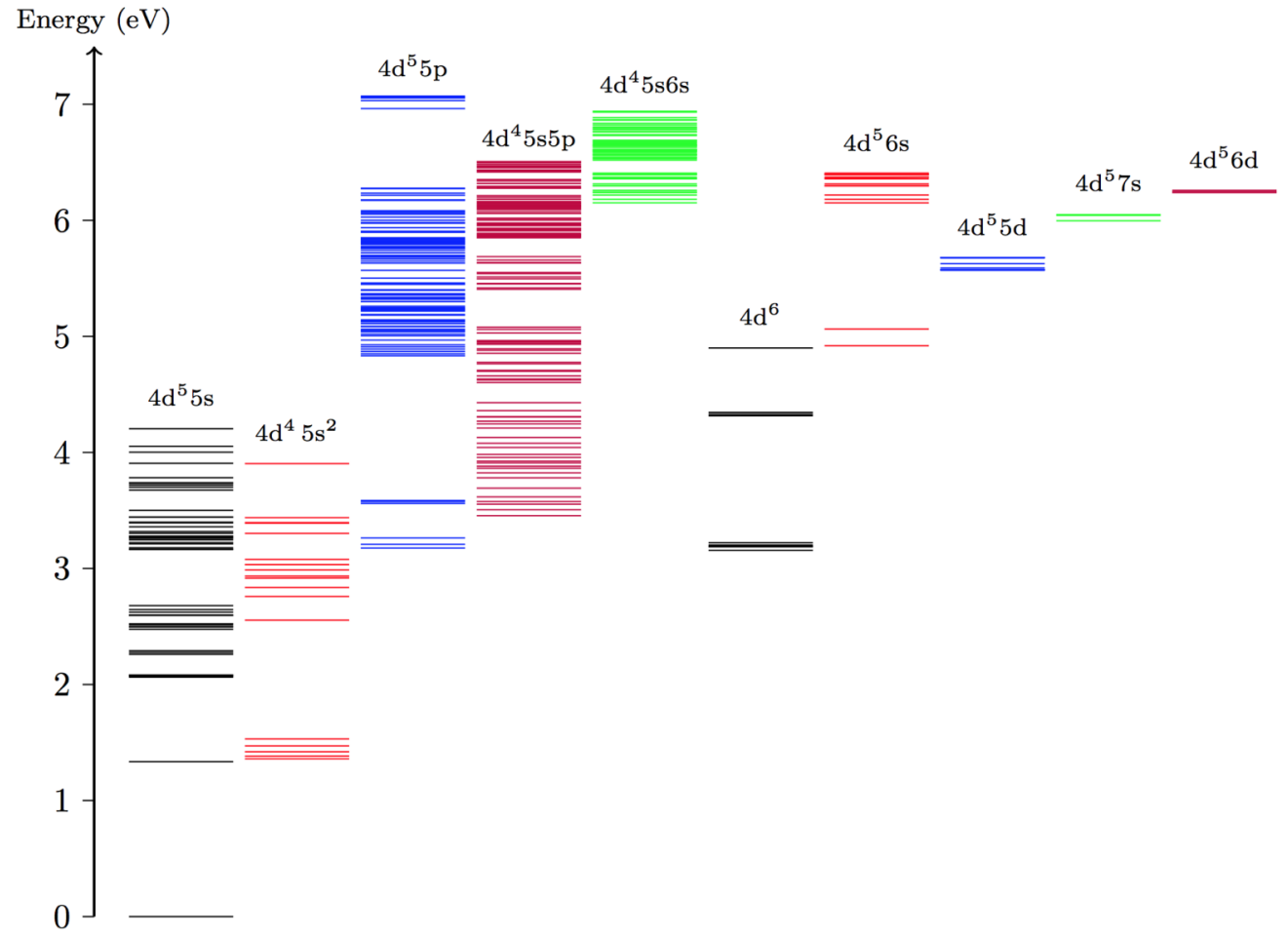

FIG. 1. Energy level spectrum of Mo I organized by electronic configuration. Each horizontal line designates a specific fine structure level listed in the NIST database.

excited states. This gives rise to hundreds of fine structure levels and thousands of coupled channels in the subsequent scattering calculations. The energy level spectrum shown in Fig. 1 illustrates the complexity of the atomic structure. Moreover, the presence of strong configuration interaction also greatly affects the difficulty of accurately modeling Mo I. A previous investigation carried out by Trees and Harvey [16] illustrates the strong mixing between states using the $a^{3} P$ and $b^{3} P$ terms and the $a^{3} D$ and $b^{3} D$ terms. This strong mixing present in Mo I proved problematic when identifying particular energy levels and transitions in our atomic structure model, especially for the highly excited states.

Our atomic structure was determined from the relativistic, multiconfigurational Dirac-Fock program GRASP ${ }^{0}$ employing a $j j$-coupling scheme for angular momentum and using a Dirac-Coulomb Hamiltonian (in atomic units) of the form

$$
H_{D}=\sum_{i}\left(-i c \boldsymbol{\alpha} \cdot \nabla_{i}+\left(\beta-I_{4}\right) c^{2}-\frac{Z}{r_{i}}\right)+\sum_{i>j} \frac{1}{r_{i j}},
$$

where $\alpha$ and $\beta$ are related to the set of Pauli spin matrices, $I_{4}$ is the $4 \times 4$ identity matrix, $Z$ is the atomic number, $c$ is the speed of light, $r_{i}$ denotes the position of electron $i$, and $r_{i j}=\left|\mathbf{r}_{i}-\mathbf{r}_{j}\right|$ is the interelectronic distance. We employed the extended average level (EAL) method, where we optimize a weighted trace of the Hamiltonian matrix using weights proportional to $(2 J+1)$, giving a reasonably good representation of closely lying states. Our model includes the following 12 nonrelativistic configurations: $4 d^{5}\{5 s, 5 p, 6 s\} ; 4 d^{4}\left\{5 s^{2}, 5 s 5 p\right.$, $\left.5 p^{2}, 6 s^{2}\right\} ; 4 d^{6} ; 4 d^{3} 5 s 6 s^{2} ; 4 p^{5} 4 d^{6} 5 s ; 4 p^{5} 4 d^{7}$; and finally $4 p^{4} 4 d^{7} 5 s$. This generated 2298 fine structure levels; however, we chose only the first 800 of these to be included in the close-coupling expansion, providing us with a comprehensive target description up to $10 \mathrm{eV}$. The significance of such an energy range is given by Badnell et al. [2], who reports on the fractional abundance of both Mo I and Mo II, showing that the abundance of Mo I is dominant below energies of approximately $10 \mathrm{eV}$, while Mo II is dominant at higher energies.

Correlating our theoretical levels with the experimental energies given in the NIST database [17] was difficult given the large amounts of mixing. However, samples of data are given in Table I. It is noted that due to the very large number of fine structure levels, only the 25 lowest even levels and 25 lowest odd levels arising from the $4 d^{5} 5 s$, $4 d^{4} 5 s^{2}, 4 d^{5} 5 p$, and $4 d^{4} 5 s 5 p$ configurations (shown clearly in Fig. 1) are reported to give an indication of the accuracy of our model.

For the odd parity levels (numbered 26-50 in Table I) we have an average percentage error of $9 \%$ between the current results and NIST values, with errors of $0.1 \%$ at best and $13 \%$ at worst. The even parity levels (numbered 1-25 in Table I) are not as well represented, having an average error of $29 \%$ and errors ranging from $3 \%$ up to $41 \%$. The largest source of error arises due to the $a^{3} D_{1}$ and $a^{3} D_{2}$ levels mentioned previously.

In Table II we present samples of some of the strongest (greater than $\left.10^{6} \mathrm{~s}^{-1}\right)$ radiative transition rates $\left(A_{j i}\right)$ obtained from our model compared to the experimental values given by Whaling et al.[18] after shifting the GRASP ${ }^{0}$ energies to experimental values. This energy shifting is carried out to ensure that our theoretically produced spectrum will yield wavelengths in agreement with experiment and is carried through to the collision calculation. We see very good agreement between the current and experimental values for 
TABLE I. Sample of Mo I fine structure energies, in Rydbergs, obtained from the GRASP ${ }^{0}$ model (relative to the ground state) and compared to the experimental values given in the NIST database. Absolute energy differences are given in the final column.

\begin{tabular}{|c|c|c|c|c|}
\hline No. & Level & NIST & GRASP $^{0}$ & $|\Delta E|$ \\
\hline 1 & $4 d^{5}\left({ }^{6} S\right) 5 s a^{7} S_{3}$ & 0.00000 & 0.00000 & 0.00000 \\
\hline 2 & $4 d^{5}\left({ }^{6} S\right) 5 \mathrm{~s} a^{5} S_{2}$ & 0.09813 & 0.10114 & 0.00301 \\
\hline 3 & $4 d^{4} 5 s^{2} a^{5} D_{0}$ & 0.09993 & 0.12035 & 0.02042 \\
\hline 4 & $4 d^{4} 5 s^{2} a^{5} D_{1}$ & 0.10154 & 0.12213 & 0.02059 \\
\hline 5 & $4 d^{4} 5 s^{2} a^{5} D_{2}$ & 0.10438 & 0.12544 & 0.02106 \\
\hline 6 & $4 d^{4} 5 s^{2} a^{5} D_{3}$ & 0.10806 & 0.12999 & 0.02193 \\
\hline 7 & $4 d^{4} 5 s^{2} a^{5} D_{4}$ & 0.11251 & 0.13559 & 0.02308 \\
\hline 8 & $4 d^{5}\left({ }^{4} G\right) 5 s a^{5} G_{2}$ & 0.15164 & 0.20967 & 0.05803 \\
\hline 9 & $4 d^{5}\left({ }^{4} G\right) 5 s a^{5} G_{3}$ & 0.15212 & 0.20996 & 0.05784 \\
\hline 10 & $4 d^{5}\left({ }^{4} G\right) 5 s a^{5} G_{4}$ & 0.15262 & 0.21027 & 0.05765 \\
\hline 11 & $4 d^{5}\left({ }^{4} G\right) 5 s a^{5} G_{6}$ & 0.15294 & 0.21059 & 0.05765 \\
\hline 12 & $4 d^{5}\left({ }^{4} G\right) 5 s \quad a^{5} G_{5}$ & 0.15295 & 0.21051 & 0.05756 \\
\hline 13 & $4 d^{5}\left({ }^{4} P\right) 5 s \quad a^{5} P_{3}$ & 0.16612 & 0.21869 & 0.05257 \\
\hline 14 & $4 d^{5}\left({ }^{4} P\right) 5 s \quad a^{5} P_{2}$ & 0.16728 & 0.21920 & 0.05192 \\
\hline 15 & $4 d^{5}\left({ }^{4} P\right) 5 s \quad a^{5} P_{1}$ & 0.16840 & 0.21966 & 0.05126 \\
\hline 16 & $4 d^{5}\left({ }^{4} D\right) 5 s b^{5} D_{0}$ & 0.18198 & 0.25173 & 0.06975 \\
\hline 17 & $4 d^{5}\left({ }^{4} D\right) 5 s b^{5} D_{1}$ & 0.18344 & 0.25217 & 0.06873 \\
\hline 18 & $4 d^{5}\left({ }^{4} D\right) 5 s b^{5} D_{4}$ & 0.18369 & 0.25291 & 0.06922 \\
\hline 19 & $4 d^{5}\left({ }^{4} D\right) 5 s b^{5} D_{2}$ & 0.18481 & 0.25290 & 0.06809 \\
\hline 20 & $4 d^{5}\left({ }^{4} D\right) 5 s b^{5} D_{3}$ & 0.18545 & 0.25337 & 0.06792 \\
\hline 21 & $4 d^{4} 5 s^{2} a^{3} P_{0}$ & 0.18779 & 0.23600 & 0.04821 \\
\hline 22 & $4 d^{5}\left({ }^{4} D\right) 5 s \quad a^{3} D_{1}$ & 0.19073 & 0.26786 & 0.07713 \\
\hline 23 & $4 d^{5}\left({ }^{4} G\right) 5 s \quad a^{3} G_{3}$ & 0.19089 & 0.24727 & 0.05638 \\
\hline 24 & $4 d^{5}\left({ }^{4} D\right) 5 s \quad a^{3} D_{2}$ & 0.19092 & 0.26926 & 0.07834 \\
\hline 25 & $4 d^{5}\left({ }^{4} G\right) 5 s a^{3} G_{4}$ & 0.19277 & 0.24798 & 0.05521 \\
\hline 26 & $4 d^{5}\left({ }^{6} S\right) 5 p z^{7} P_{2}^{o}$ & 0.23342 & 0.21244 & 0.02098 \\
\hline 27 & $4 d^{5}\left({ }^{6} S\right) 5 p \quad z^{7} P_{3}^{o}$ & 0.23576 & 0.21465 & 0.02111 \\
\hline 28 & $4 d^{5}\left({ }^{6} S\right) 5 p z^{7} P_{4}^{o}$ & 0.23985 & 0.21760 & 0.02225 \\
\hline 29 & $4 d^{4} 5 s\left({ }^{6} D\right) 5 p \quad z^{7} F_{0}^{o}$ & 0.25394 & 0.22294 & 0.03100 \\
\hline 30 & $4 d^{4} 5 s\left({ }^{6} D\right) 5 p \quad z^{7} F_{1}^{o}$ & 0.25765 & 0.22420 & 0.03345 \\
\hline 31 & $4 d^{4} 5 s\left({ }^{6} D\right) 5 p \quad z^{7} F_{2}^{o}$ & 0.26123 & 0.22668 & 0.03455 \\
\hline 32 & $4 d^{5}\left({ }^{6} S\right) 5 p \quad z^{5} P_{1}^{o}$ & 0.26167 & 0.27037 & 0.00870 \\
\hline 33 & $4 d^{5}\left({ }^{6} S\right) 5 p \quad z^{5} P_{2}^{o}$ & 0.26278 & 0.27116 & 0.00838 \\
\hline 34 & $4 d^{4} 5 s\left({ }^{6} D\right) 5 p \quad z^{7} F_{3}^{o}$ & 0.26288 & 0.23033 & 0.03255 \\
\hline 35 & $4 d^{5}\left({ }^{6} S\right) 5 p \quad z^{5} P_{3}^{o}$ & 0.26357 & 0.27226 & 0.00869 \\
\hline 36 & $4 d^{4} 5 s\left({ }^{6} D\right) 5 p \quad z^{7} F_{4}^{o}$ & 0.26583 & 0.23507 & 0.03076 \\
\hline 37 & $4 d^{4} 5 s\left({ }^{6} D\right) 5 p \quad z^{7} F_{5}^{o}$ & 0.27139 & 0.24086 & 0.03053 \\
\hline 38 & $4 d^{4} 5 s\left({ }^{6} D\right) 5 p \quad z^{7} F_{6}^{o}$ & 0.27790 & 0.24764 & 0.03026 \\
\hline 39 & $4 d^{4} 5 s\left({ }^{6} D\right) 5 p \quad z^{7} D_{1}^{o}$ & 0.28109 & 0.25073 & 0.03036 \\
\hline 40 & $4 d^{4} 5 s\left({ }^{6} D\right) 5 p \quad z^{7} D_{2}^{o}$ & 0.28390 & 0.25352 & 0.03038 \\
\hline 41 & $4 d^{4} 5 s\left({ }^{6} D\right) 5 p \quad y^{7} P_{2}^{o}$ & 0.28523 & 0.26174 & 0.02349 \\
\hline 42 & $4 d^{4} 5 s\left({ }^{6} D\right) 5 p \quad y^{7} P_{3}^{o}$ & 0.28735 & 0.26435 & 0.02300 \\
\hline 43 & $4 d^{4} 5 s\left({ }^{6} D\right) 5 p \quad z^{7} D_{3}^{o}$ & 0.28846 & 0.25731 & 0.03115 \\
\hline 44 & $4 d^{4} 5 s\left({ }^{6} D\right) 5 p \quad y^{7} P_{4}^{o}$ & 0.29081 & 0.26785 & 0.02296 \\
\hline 45 & $4 d^{4} 5 s\left({ }^{6} D\right) 5 p \quad z^{7} D_{4}^{o}$ & 0.29273 & 0.26185 & 0.03088 \\
\hline 46 & $4 d^{4} 5 s\left({ }^{6} D\right) 5 p \quad z^{7} D_{5}^{o}$ & 0.29718 & 0.26695 & 0.03023 \\
\hline 47 & $4 d^{4} 5 s\left({ }^{6} D\right) 5 p \quad y^{5} P_{1}^{o}$ & 0.29980 & 0.29720 & 0.00260 \\
\hline 48 & $4 d^{4} 5 s\left({ }^{6} D\right) 5 p \quad y^{5} P_{2}^{o}$ & 0.30344 & 0.30200 & 0.00144 \\
\hline 49 & $4 d^{4} 5 s\left({ }^{6} D\right) 5 p \quad y^{5} P_{3}^{o}$ & 0.30942 & 0.30918 & 0.00024 \\
\hline 50 & $4 d^{4} 5 s\left({ }^{6} D\right) 5 p \quad z^{5} F_{1}^{o}$ & 0.31209 & 0.31148 & 0.00061 \\
\hline
\end{tabular}

TABLE II. The radiative transition rates (in $\mathrm{s}^{-1}$ ) obtained from the present model compared to experimental values given in Ref. [18]. The rates shown are obtained after shifting energies to experimental values.

\begin{tabular}{|c|c|c|c|}
\hline \multirow[b]{2}{*}{$\lambda(\AA)$} & \multirow{2}{*}{$\begin{array}{c}\text { Transition } \\
\quad j-i\end{array}$} & \multicolumn{2}{|c|}{$A_{j i}\left(s^{-1}\right)$} \\
\hline & & Current & Whaling \\
\hline 3132.59 & $44-1$ & $2.88 \times 10^{8}$ & $1.78 \times 10^{8}$ \\
\hline 3170.34 & $42-1$ & $2.76 \times 10^{8}$ & $1.36 \times 10^{8}$ \\
\hline 3193.97 & $41-1$ & $2.77 \times 10^{8}$ & $1.53 \times 10^{8}$ \\
\hline 3456.39 & $35-1$ & $4.93 \times 10^{6}$ & $4.20 \times 10^{6}$ \\
\hline 3466.82 & $33-1$ & $2.63 \times 10^{6}$ & $1.23 \times 10^{6}$ \\
\hline 3798.26 & $28-1$ & $7.56 \times 10^{7}$ & $6.90 \times 10^{7}$ \\
\hline 3864.11 & $27-1$ & $6.95 \times 10^{7}$ & $6.20 \times 10^{7}$ \\
\hline 3902.96 & $26-1$ & $6.55 \times 10^{7}$ & $6.17 \times 10^{7}$ \\
\hline 4293.87 & $50-3$ & $3.74 \times 10^{6}$ & $5.90 \times 10^{6}$ \\
\hline 4326.74 & $49-4$ & $3.19 \times 10^{6}$ & $4.50 \times 10^{6}$ \\
\hline 4512.13 & $48-4$ & $9.64 \times 10^{5}$ & $1.00 \times 10^{6}$ \\
\hline 4524.33 & $49-6$ & $2.42 \times 10^{6}$ & $1.76 \times 10^{6}$ \\
\hline 4558.10 & $47-3$ & $1.46 \times 10^{6}$ & $1.14 \times 10^{6}$ \\
\hline 4576.49 & $48-5$ & $3.10 \times 10^{6}$ & $2.14 \times 10^{6}$ \\
\hline 4594.32 & $47-4$ & $2.96 \times 10^{6}$ & $1.90 \times 10^{6}$ \\
\hline 4626.45 & $49-7$ & $6.62 \times 10^{6}$ & $4.00 \times 10^{6}$ \\
\hline 4662.75 & $48-6$ & $3.73 \times 10^{6}$ & $2.06 \times 10^{6}$ \\
\hline 4979.12 & $39-2$ & $1.38 \times 10^{6}$ & $2.30 \times 10^{6}$ \\
\hline 5506.50 & $35-2$ & $5.41 \times 10^{7}$ & $3.42 \times 10^{7}$ \\
\hline 5533.03 & $33-2$ & $5.47 \times 10^{7}$ & $3.59 \times 10^{7}$ \\
\hline 5570.43 & $32-2$ & $5.39 \times 10^{7}$ & $3.23 \times 10^{7}$ \\
\hline 5632.46 & $32-3$ & $3.18 \times 10^{6}$ & $7.16 \times 10^{6}$ \\
\hline 5689.14 & $32-4$ & $6.72 \times 10^{6}$ & $1.45 \times 10^{7}$ \\
\hline 5650.14 & $33-4$ & $1.41 \times 10^{6}$ & $1.58 \times 10^{6}$ \\
\hline 5677.87 & $50-8$ & $3.05 \times 10^{5}$ & $1.39 \times 10^{6}$ \\
\hline 5689.14 & $32-4$ & $6.72 \times 10^{6}$ & $1.45 \times 10^{7}$ \\
\hline 5722.76 & $35-5$ & $4.66 \times 10^{5}$ & $1.94 \times 10^{6}$ \\
\hline 5751.42 & $33-5$ & $4.76 \times 10^{6}$ & $4.30 \times 10^{6}$ \\
\hline 5791.85 & $32-5$ & $4.82 \times 10^{6}$ & $1.26 \times 10^{7}$ \\
\hline 5858.28 & $35-6$ & $2.57 \times 10^{6}$ & $5.93 \times 10^{6}$ \\
\hline 5888.32 & $33-6$ & $6.78 \times 10^{6}$ & $7.30 \times 10^{6}$ \\
\hline
\end{tabular}

the $z^{7} P_{2,3,4}^{o} \rightarrow a^{7} S_{3}$ (labeled 26,27,28 $\rightarrow 1$ in Table II) with an average percentage difference of $9 \%$. The $z^{5} P_{1,2,3}^{o} \rightarrow a^{5} S_{2}$ transitions (labeled $32,33,35 \rightarrow 2$ ) agree reasonably well, having an average difference of $45 \%$. Finally, although the $y^{7} P_{2,3,4}^{o} \rightarrow a^{7} S_{3}$ (labeled $41,42,44 \rightarrow 1$ ) are not as well represented, having an average difference of 58\%, they are still in reasonable agreement with experimental values. Considering all transitions yields an average percentage difference of $47 \%$. These transitions of experimental interest are illustrated clearly in Fig. 2.

An assessment of the accuracy of this model in relation to previous calculations will be presented alongside the results of the $R$-matrix scattering calculation in Sec. III C.

\section{ELECTRON-IMPACT EXCITATION}

\section{A. $R$-matrix theory}

Within the framework of $R$-matrix theory [19] we partition configuration space into two distinct regions, an inner region and an outer region, separated by an $R$-matrix boundary at 


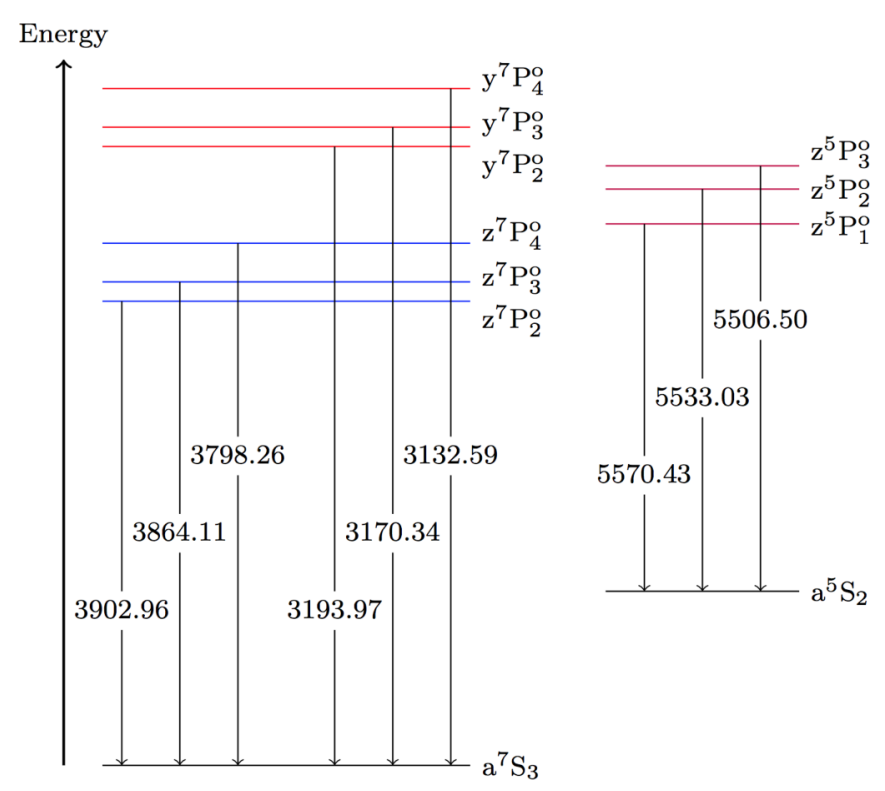

FIG. 2. An illustration showing the fine structure transitions (with wavelengths in $\AA$ ) of experimental interest.

$r=a$, chosen as to completely enclose the charge distribution of the $N$-electron target. We therefore require that the effects of correlation, exchange, and electron capture to be accounted for within the inner region which allows us to neglect these effects within the outer region, where the electron only moves in the long-range potential of the $N$-electron target.

The inner region wave function of the $N+1$ electron system (that is, the $N$ electron target plus an addition electron) is expanded in terms of an energy-independent basis such that

$$
\Psi_{j E}^{\Gamma}\left(\mathbf{X}_{N+1}\right)=\sum_{k} \psi_{k}^{\Gamma}\left(\mathbf{X}_{N+1}\right) A_{j k}^{\Gamma}(E),
$$

where $A_{j k}^{\Gamma}(E)$ are energy-dependent coefficients and the energy-independent basis functions are given by

$$
\begin{aligned}
\psi_{k}^{\Gamma}= & \mathcal{A} \sum_{i} \sum_{j} \bar{\Phi}_{i}^{\Gamma}\left(\mathbf{X}_{N} ; \hat{\mathbf{r}}_{N+1} \sigma_{N+1}\right) r_{N+1}^{-1} u_{i j}\left(r_{N+1}\right) a_{i j k}^{\Gamma} \\
& +\sum_{i} \chi_{i}^{\Gamma}\left(\mathbf{X}_{N+1}\right) b_{i k}^{\Gamma},
\end{aligned}
$$

where $\Gamma=J M_{J} \pi$ is the set of conserved quantum numbers, $\mathbf{X}_{N+1}=\mathbf{x}_{1}, \ldots, \mathbf{x}_{N+1}$ (where $\left.\mathbf{x}_{i}=\mathbf{r}_{i} \sigma_{i}\right)$ are the set of space and spin coordinates of the $N+1$ electrons, $\bar{\Phi}_{i}^{\Gamma}$ are channel functions, $u_{i j}$ are continuum basis functions for the scattered electron, $\chi_{i}^{\Gamma}$ are square integrable functions, and the coefficients $a_{i j k}^{\Gamma}$ and $b_{i k}^{\Gamma}$ are determined from a single diagonalization of the $N+1$ electron Hamiltonian [analogous to Eq. (1)] in the basis defined by Eq. (3). The total wave function in the outer region is given by

$$
\Psi_{j E}^{\Gamma}=\sum_{i} \bar{\Phi}_{i}^{\Gamma}\left(\mathbf{X}_{N} ; \hat{\mathbf{r}}_{N+1} \sigma_{N+1}\right) r_{N+1}^{-1} F_{i j}^{\Gamma}\left(r_{N+1}\right),
$$

where $\bar{\Phi}_{i}^{\Gamma}$ are the same channel functions as in Eq. (3) and $F_{i j}^{\Gamma}$ are reduced radial functions. Matching the form of the outer region wave function to asymptotic boundary conditions given by Young and Norrington [20] allows one to determine the collision strengths $\left(\Omega_{i f}\right)$ of the excitation and, by extension, the excitation cross section for a transition from some initial state $i$ to a final state $f$, defined (in units of $\mathrm{Mb}=10^{-22} \mathrm{~m}^{2}$ ) as

$$
\sigma_{i \rightarrow f}=\frac{\Omega_{i f}}{k_{i}^{2}\left(2 J_{i}+1\right)},
$$

where $J_{i}$ is the total angular momentum of the initial state, $k_{i}^{2}=2 \epsilon_{i}$, and $\epsilon_{i}$ is the scattered electron energy in the $i$ th channel, in Rydbergs.

\section{B. Scattering calculation}

The electron-impact excitation calculations for Mo I were carried out with the parallel suite of fully relativistic $R$-matrix codes (DARC) using the GRASP ${ }^{0}$ model as the $N$-electron target. As mentioned in Sec. II, 800 levels out of the 2298 level GRASP $^{0}$ structure were retained in the scattering calculation, resulting in a very computationally demanding model that required thousands of cores on large supercomputing facilities. The $R$-matrix boundary was set at 29.76 atomic units, and 15 continuum basis orbitals were used for each value of angular momentum $J$. Target energies were shifted to the experimental values given in the NIST database, as mentioned in Sec. II, by adjusting the diagonal elements of the target Hamiltonian ensuring wavelengths are in agreement with experiment. Calculations were performed for $60 J \pi$ partial waves from $2 J=1$ up to $2 J=59$ giving Hamiltonian matrices of sizes up to $86250 \times 86250$ and generated up to 5750 coupled channels.

For low $J \pi$ partial waves from $2 J=1$ up to $2 J=25$, a mesh of 10000 energy points with an energy spacing of $6.41 \times 10^{-5}$ Ryd was used. For higher partial waves from $2 J=27$ up to $2 J=59$ a much coarser mesh of 250 points with energy spacing $2.56 \times 10^{-3}$ Ryd was used. A "top-up" procedure described by Burgess [21] was then employed to estimate the contributions of partial waves $2 J>59$. This mesh provides an energy range of approximately $0-10 \mathrm{eV}$. An additional 300 energy points were used to span the 10-20 eV range, with an energy spacing of $2.47 \times 10^{-3} \mathrm{Ryd}$, used for all partial waves $2 J=1$ up to $2 J=59$, again with the "top-up" procedure employed for $2 J>59$.

\section{Results and discussion}

In Figs. 3-5 we present excitation cross sections (in units of $\mathrm{Mb}$ ) from this 800 state, fully relativistic $R$-matrix scattering calculation. These are compared with the results of the two previous calculations in $L S$ coupling, for which we must statistically average our fine structure results. We focus on the $a^{7} S \rightarrow z^{7} P^{o}, a^{7} S \rightarrow y^{7} P^{o}$, and $a^{5} S \rightarrow z^{5} P^{o}$ electric dipole transitions of interest to diagnostic work. One comparison will be made with the results of Badnell et al.[2], who carried out nonrelativistic 3 state and 6 state $R$-matrix calculations for the dipole and intercombination transitions, respectively, using only the $a^{7} S, a{ }^{5} S, z^{7} P^{o}, z^{5} P^{o}, y^{7} P^{o}$, and $y^{5} P^{o}$ terms. The other comparison will be with the work of Bartschat et al., [3] who carried out a nonrelativistic 67 state $R$-matrix calculation including only septet, quintet, and triplet terms below $7 \mathrm{eV}$.

It is evident from Fig. 3 that our excitation cross section for the $a^{7} S \rightarrow z^{7} P^{o}$ excitation is smaller than those presented by both [2] and [3]. However, we see good agreement in shape 


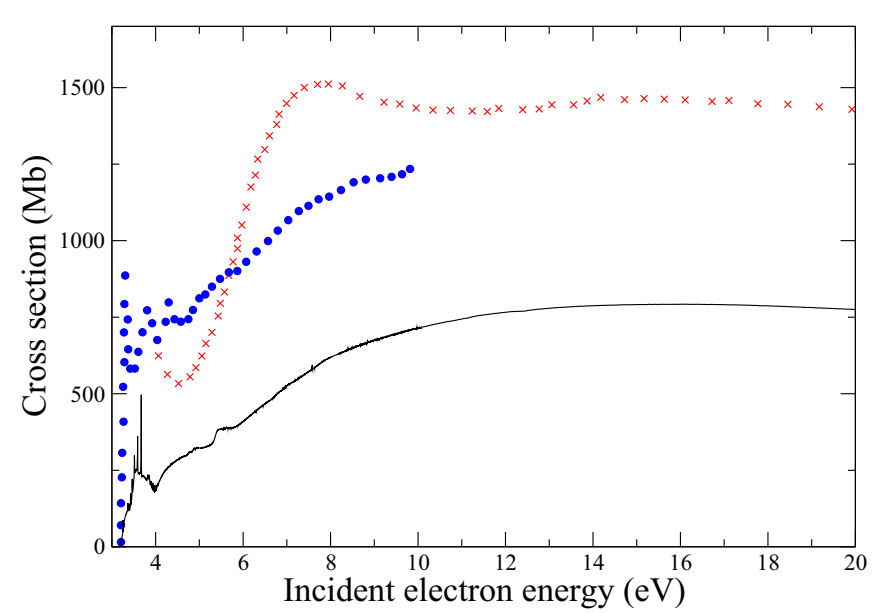

FIG. 3. The $a^{7} S \rightarrow z^{7} P^{o}$ excitation cross section in Mb. The black curve is the current $R$-matrix result, red crosses are the results of [2], and blue circles are the results of [3].

between our cross section and the one presented by [3]. These variations potentially arise due to differences in the target structures used and the specific details of the scattering calculations. We compare the transition rate (in $L S$ coupling) from our atomic structure calculation (after statistically averaging the fine structure values in Table II) with the value given by [2] (after shifting the reported rates to experimental energy differences), the value given by [3], and the experimental value given by Whaling et al.[18] (again, after statistically averaging the fine structure values). Our GRASP ${ }^{0}$ structure gives an $A$-value of $7.12 \times 10^{7} \mathrm{~s}^{-1}$ compared to the experimental value of $6.49 \times 10^{7} \mathrm{~s}^{-1}$, and the values of $2.01 \times 10^{8} \mathrm{~s}^{-1}$ and $1.62 \times 10^{8} \mathrm{~s}^{-1}$ reported by [2] and [3], respectively. We see very good agreement with the experimental result of [18].

We see from Fig. 4 that our excitation cross section for the $a^{7} S \rightarrow y^{7} P^{o}$ excitation agrees reasonably well with that of [2] up to approximately $10 \mathrm{eV}$, after which they begin to diverge. In contrast, no agreement is seen with the cross section presented by [3]. Again, we look at differences in the targets used. The current GRASP ${ }^{0}$ model gives a spontaneous emission

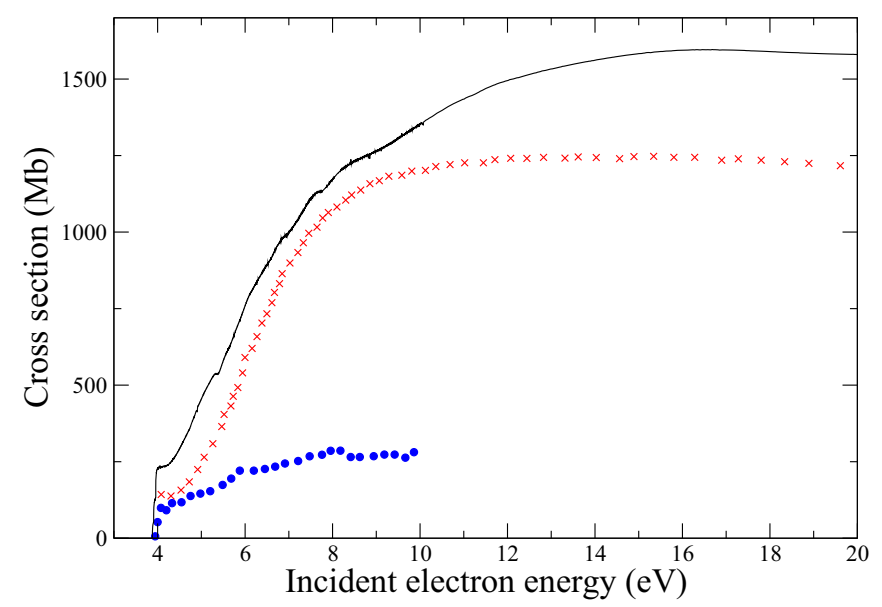

FIG. 4. The $a^{7} S \rightarrow y^{7} P^{o}$ excitation cross section in Mb. The black curve is the current $R$-matrix result, red crosses are the results of [2], and blue circles are the results of [3].

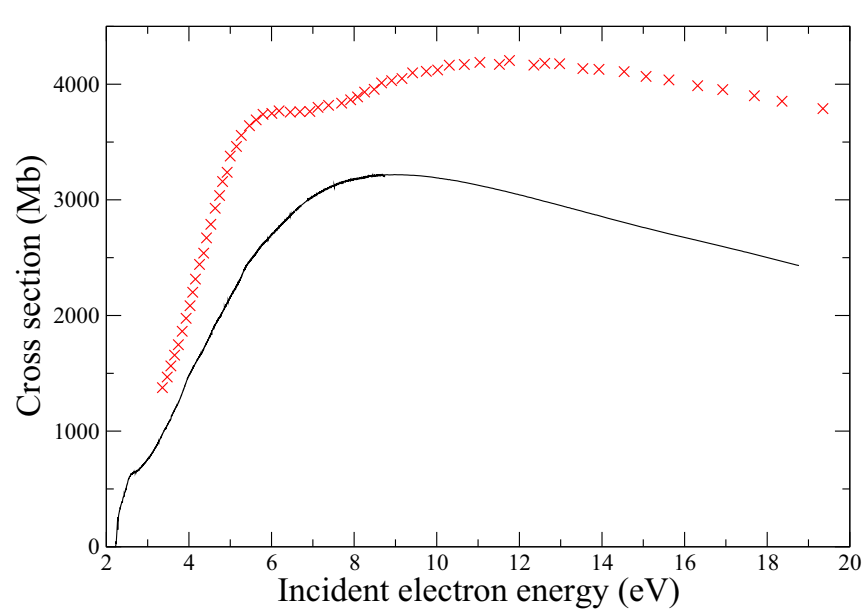

FIG. 5. The $a^{5} S \rightarrow z^{5} P^{o}$ excitation cross section in Mb. The black curve is the current $R$-matrix result and red crosses are the results of [2].

rate of $2.81 \times 10^{8} \mathrm{~s}^{-1}$ compared to the experimental value of $1.58 \times 10^{8} \mathrm{~s}^{-1}$, and values of $1.88 \times 10^{8} \mathrm{~s}^{-1}$ and $0.73 \times$ $10^{8} \mathrm{~s}^{-1}$ reported by [2] and [3], respectively. It is clear that the small cross section presented by [3] is consistent with their $A$-value being lower than the experimental value.

Finally, in Fig. 5, it is evident that our cross section for the $a^{5} S \rightarrow z^{5} P^{o}$ excitation falls below the one presented by [2]. Our GRASP ${ }^{0}$ model yields an $A$-value of $5.42 \times 10^{7} \mathrm{~s}^{-1}$ compared to the experimental value of $3.44 \times 10^{7} \mathrm{~s}^{-1}$, these in comparison with the value of $1.64 \times 10^{8} \mathrm{~s}^{-1}$ reported by [2]. Again, good agreement between the radiative transition rates can be seen with the experimental result of [18]. No excitation cross section or transition rate is reported by [3] and thus no comparison can be made.

\section{MOLYBDENUM SPECTRA}

\section{A. Experiment}

As an illustration of the use of the new atomic data, spectral measurements of Mo emission were acquired with the Compact Toroidal Hybrid plasma experiment (CTH) at Auburn University [1]. A vertically translating probe with a Mo tip was constructed and inserted into the edge of CTH plasmas with an optical line of sight observing emission from the Mo-plasma interaction. Stellarnet survey spectrometers sensitive between $300-400 \mathrm{~nm}$ and 400-600 nm are coupled by an optical fiber to collection optics mounted opposite to the translating probe and focused on the Mo probe tip. While the CTH plasma parameters depend on the specific operating conditions, the electron density and temperature of the plasma in the region around the Mo probe tip for the measurements shown herein are expected to be $T_{\mathrm{e}}$ from 1 to $10 \mathrm{eV}$ and $n_{e} \approx 10^{12} \mathrm{~cm}^{-3}$. Observed plasma-Mo emission spectra are shown for the Mo I triplet sets listed as (i) and (ii) in the Introduction, see Fig. 6.

\section{B. Modeling}

Using the collision strengths $\left(\Omega_{i f}\right)$ obtained from the $R$-matrix scattering calculation and the radiative transition rates $\left(A_{j i}\right)$ from the atomic structure calculation we construct 

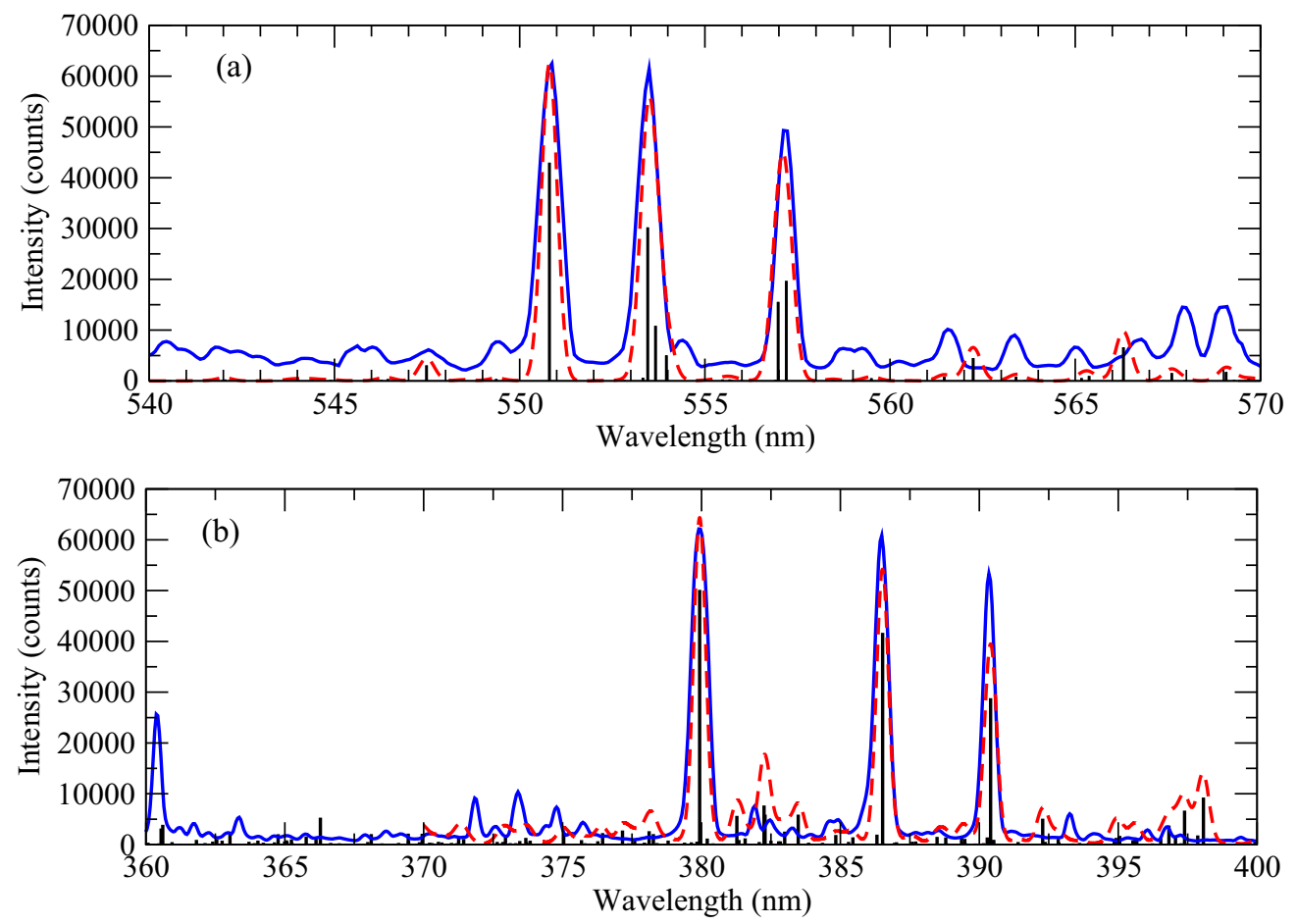

FIG. 6. Measured spectrum from the CTH plasma (solid blue line), compared with theoretical results. The solid black sticks show the PEC coefficients for the Mo I transitions, while the dashed red curve shows a theoretical spectrum based upon Gaussian convolved PEC data. A FWHM for the Gaussian convolution of $0.15 \mathrm{~nm}$ was used, based upon the instrument resolution, and the PECs are shown for an electron temperature of $6 \mathrm{eV}$ and an electron density of $1 \times 10^{12} \mathrm{~cm}^{-3}$.

a ground state resolved collisional-radiative model using collisional-radiative theory [22] to determine the populations of the excited levels $\bar{N}_{j}=N_{j} / N_{1}$, normalized to the ground state. In turn, these populations allow the calculation of the photon emissivity coefficients (PECs) (in units of number of photons $\mathrm{cm}^{3} \mathrm{~s}^{-1}$ ) defined as

$$
\mathcal{P} \mathcal{E} \mathcal{C}_{1, j \rightarrow i}^{(\mathrm{exc})}=A_{j i} \mathcal{F}_{j, 1}^{(\mathrm{exc})}=-A_{j i} \sum_{k>1}\left(C_{j k}^{\prime}\right)^{-1} C_{k 1} .
$$

Here, $\mathcal{F}_{j, 1}^{(\text {exc })}$ is the effective contribution to excited level $j$ through collisional excitation from the ground level, $C_{i j}$ is the collisional-radiative matrix, obtained from balancing the excitation and radiative decay rates, and $C_{i j}^{\prime}$ is the reduced collisional-radiative matrix with the ground state row removed. Further details on the collisional-radiative equations can be found in Ref. [23].

In Fig. 6 we show a plot of the PECs calculated using our structure and collision data, covering the lines in the 379$391 \mathrm{~nm}$ range and the 550-558 $\mathrm{nm}$ range and for the electron temperature and density values expected in CTH in the region of the Mo probe. We compare with a spectrum obtained from the CTH experiment, as described in the previous section. It is clear that there is very good agreement with the strongest $z^{7} P_{2,3,4}^{o} \rightarrow a^{7} S_{3}$ and $z^{5} P_{1,2,3}^{o} \rightarrow a^{5} S_{2}$ transitions. This high level of agreement gives credence to the accuracy of the present atomic structure data. It must be noted that additional peaks are seen in the experimental spectrum due to the presence of impurities within the plasma. A full comparison of the CTH spectrum and the new theoretical data will be the subject of future work and will include investigations of the other Mo I lines that were observed, the sensitivity of the lines to plasma electron temperature and density, and the role of metastable states. The full set of data used here will also be made available on the OPEN-ADAS site [24].

\section{CONCLUSIONS}

In this work we have carried out extensive atomic structure and electron-impact excitation calculations for neutral molybdenum. We have highlighted the incomplete nature of the two existing models and improved upon these, helping to rectify the current discrepancies between theory and experiment.

The atomic structure was investigated using the relativistic multiconfigurational Dirac-Fock method and results are compared to available experimental data, with a particular focus on the transitions of spectroscopic interest. The atomic structure was then carried through to an 800 state relativistic $R$-matrix scattering calculation, and excitation cross sections are compared to the results of two available theoretical models. Comparisons show large differences between the new and previous cross sections, highlighting the deficiencies contained within the previous models.

Collisional-radiative theory was employed to calculate photon emissivity coefficients which allowed us to validate and benchmark the new atomic structure and excitation results with experimental data obtained from a recent CTH experiment, showing good agreement across a wide wavelength range. We have identified the strongest $z^{7} P_{2,3,4}^{o} \rightarrow a^{7} S_{3}$ and $z^{5} P_{1,2,3}^{o} \rightarrow$ $a^{5} S_{2}$ lines that will be useful for diagnostic work, the radiative transition rates for which are in better agreement with experimental values than previous calculations. In addition, given 
the high level of accuracy, we believe that the $y{ }^{7} P_{2,3,4}^{o} \rightarrow a{ }^{7} S_{3}$ lines will also be useful for diagnostic work.

The data presented in this study, coupled with new $R$-matrix electron-impact ionization calculations, will be used in further work to diagnose the influx of high- $Z$ impurities into magnetically confined fusion plasmas and will be useful for other applications of neutral molybdenum requiring structure and collision data of high accuracy.

\section{ACKNOWLEDGMENTS}

R. T. Smyth is funded by the STFC ST/P000312/1 - QUB Astronomy Observation and Theory Consolidated Grant. All calculations were carried out at the Cray XC40 "Hazelhen" supercomputer in HLRS Stuttgart, the Cray XC40 "Cori" supercomputer at NERSC California, and a local cluster at Queen's University Belfast.
[1] G. J. Hartwell, S. F. Knowlton, J. D. Hanson, D. A. Ennis, and D. A. Maurer, Fusion Sci. Technol. 72, 76 (2017).

[2] N. R. Badnell, T. W. Gorczyca, M. S. Pindzola, and H. P. Summers, J. Phys. B 29, 3683 (1996).

[3] K. Bartschat, A. Dasgupta, and J. L. Giuliani, J. Phys. B 35, 2899 (2002).

[4] A. N. James, D. Brunner, B. Labombard, C. Lau, B. Lipschultz, D. Miller, M. L. Reinke, J. L. Terry, C. Theiler, G. M. Wallace et al., Plasma Phys. Controlled Fusion 55, 125010 (2013).

[5] B. Lipschultz, D. Pappas, B. LaBombard, J. Rice, D. Smith, and S. Wukitch, Nucl. Fusion 41, 585 (2001).

[6] D. Pappas, B. Lipschultz, B. LaBombard, M. May, and C. Pitcher, J. Nucl. Mater. 266-269, 635 (1999).

[7] D. Nishijima, R. P. Doerner, D. G. Whyte, M. J. Baldwin, and T. Schwarz-Selinger, J. Phys. B 43, 225701 (2010).

[8] R. Boivin, J. Brooks, M. Khandagle, Y. Hirooka, and J. Won, J. Nucl. Mater. 230, 101 (1996).

[9] W. Wampler, P. Stangeby, J. Watkins, D. Buchenauer, D. Rudakov, and C. Wong, Proceedings of the 20th International Conference on Plasma-Surface Interactions in Controlled Fusion Devices, edited by S. Brezinsek, A. Kreter, V. Philipps, G. Pintsuk, D. Reiter, and B. Unterberg, Vol. 438 (Elsevier B.V., Aachen, 2013).

[10] F. Scotti, V. A. Soukhanovskii, and M. E. Weller, Rev. Sci. Instrum. 87, 11D445 (2016).
[11] J. Wesson, Tokamaks (Oxford, University Press, Oxford, UK, 1987).

[12] K. Behringer, H. P. Summers, B. Denne, M. Forrest, and M. Stamp, Plasma Phys. Controlled Fusion 31, 2059 (1989).

[13] F. A. Parpia and I. P. Grant, J. Phys. IV (France) 01, C1 (1991).

[14] K. Dyall, I. Grant, C. Johnson, F. Parpia, and E. Plummer, Comput. Phys. Commun. 55, 425 (1989).

[15] DARC, http://connorb.freeshell.org/

[16] R. E. Trees and M. M. Harvey, J. Res. Natl. Bur. Stand. 49, 379 (1952).

[17] NIST, http://www.nist.gov/pml/data/asd.cfm

[18] W. Whaling, P. Hannaford, R. Lowe, E. Biémont, and N. Grevesse, J. Quant. Spectrosc. Radiat. Transfer 32, 69 (1984).

[19] P. Burke, R-Matrix Theory of Atomic Collisions: Application to Atomic, Molecular and Optical Processes, Springer Series on Atomic, Optical, and Plasma Physics (Springer, Berlin, 2011).

[20] I. Young and P. Norrington, Comput. Phys. Commun. 83, 215 (1994).

[21] A. Burgess, J. Phys. B 7, L364 (1974).

[22] H. P. Summers, W. J. Dickson, M. G. O’Mullane, N. R. Badnell, A. D. Whiteford, D. H. Brooks, J. Lang, S. D. Loch, and D. C. Griffin, Plasma Phys. Controlled Fusion 48, 263 (2006).

[23] D. R. Bates, A. E. Kingston, and R. W. P. McWhirter, Proc. R. Soc. London A 267, 297 (1962).

[24] OPEN-ADAS, http://open.adas.ac.uk/ 